\title{
Mitarbeiter dieser Auflage
}

Die im folgenden aufgeführten Wissenschaftlerinnen und Wissenschaftler waren - in Zusammenarbeit mit der Wörterbuchredaktion des Verlages - an der Abfassung von Texten beteiligt, die in dieser Ausgabe des Wörterbuchs veröffentlicht werden.

Priv.-Doz. Dr. med. Fuat Aksu

Ltd. Oberarzt der Klinik für Neuropädiatrie der Medizinischen Universität zu Lübeck Kahlhorststraße 31-35

2400 Lübeck 1

Dr. med. Gisela Albrecht Chefärztin der Abt. Dermatologie Krankenhaus Spandau Nord Lynarstraße 12 1000 Berlin 20

Dr. med. Elke Austenat

Ärztin für Innere Medizin

Dudenstraße 6

1000 Berlin 61

Dr. rer, nat. Dipl.-Chem. Lutz Austenat

GSD Gesellschaft für Systemforschung und Dienstleistungen im Gesundheitswesen $\mathrm{mbH}$ Stauffenbergstr. 13-14 1000 Berlin 30

Dr. med. Klaus Badenhoop

II. Medizinische Klinik, Klinikum Mannheim der Universität Heidelberg

Theodor-Kutzer-Ufer

6800 Mannheim 1

Prof. Dr. med. Leonore Ballowitz

Havelmatensteig 12

1000 Berlin 22

Priv.-Doz. Dr. med. Dietrich Banzer

Chefarzt der Röntgen- und Nuklearmedizinischen Abt. des Krankenhauses Berlin-

Zehlendorf (Behring-Krankenhaus)

Gimpelsteig 3

1000 Berlin 37

Dr. med. Hans-Helge Bartsch

Abt. Hämatologie/Onkologie

Zentrum Innere Medizin

der Georg-August-Universität

Robert-Koch-Str. 40

3400 Göttingen

Prof. Dr. med. Rolf Baß

Leiter der Abt. Toxikologie

Institut für Arzneimittel des Bundesgesund-

heitsamtes,

Seestraße 10

1000 Berlin 65
Prof. Dr. Dr. med. habil. H. W. Bauer

Maximilianstraße 31

8000 München 22

Univ.-Prof. Dr. med. Hans-Peter Berlien

Leiter des Fachgebietes Lasermedizin

Klinikum Steglitz der FU Berlin

Med. Direktor des Laser-Medizin-Zentrums

Krahmerstr. 6-10

1000 Berlin 45

Renate Beuerle

Ârztin

Kreiskrankenhaus Lehrte

Manskestr. 22

3160 Lehrte

Prof. Dr. med. H. J. Biersack

Institut für Klinische und Experimentelle

Nuklearmedizin der Universität Bonn

Sigmund-Freud-Str. 25

5300 Bonn 1

Dipl.-Phys. Dieter Borchardt

Hahn-Meitner-Institut für Kernforschung

Berlin GmbH, Abt. Strahlenschutz

Glienicker Str. 100

1000 Berlin 39

Dr. med. Dieter Borgers, MPH

IDIS Institut für Dokumentation und

Information, Sozialmedizin und öffentliches

Gesundheitswesen

Westerfeldstr. 35-37

4800 Bielefeld 1

\section{R.-D. Bredtmann}

Arzt für Anästhesiologie

Anästhesieabteilung des Allgemeinen

Krankenhauses Altona

Paul-Ehrlich-Str. 1

2000 Hamburg 50

Prof. Dr. phil. Mag. pharm. Artur Burger

Institut für Pharmakognosie

der Universität Innsbruck

Innrain 52

A - 6020 Innsbruck

Dr. med. Hans-Jürgen Christen

Kinderklinik der Georg-August-Universität

Robert-Koch-Str. 40

3400 Göttingen 
Prof. Dr. med. O. Creutzfeldt

Max-Planck-Institut für Biophysikalische Chemie

Karl Friedrich-Bonhoeffer-Institut

Postfach 2841

3400 Göttingen

Prof. Dr. med. Gisela Dallenbach-Hellweg Institut für Pathologie

A2,2

6800 Mannheim 1

Chefarzt Priv.-Doz. Dr. med. H. Dancygier Städtische Kliniken Offenbach/Main

Medizinische Klinik II

Starkenburgring 66

6050 Offenbach/Main

Dr. med. Hans Joachim Dietrich

Neurologische Klinik und Poliklinik der

Universität Erlangen-Nürnberg

Schwabachanlage 6

8520 Erlangen

Herbert Dihlmann
Arzt
Dürerplatz 3
1000 Berlin 41

Prof. Dr. med. Gerhard Döring

Seeleite 9

8031 Seefeld 2

Prof. Dr. med. Fritz Dressler

Dernburgstr. 26

1000 Berlin 19

Dr. med. Dr. phil. Stephan Dressler

Durlacher Str. 23

1000 Berlin 31

Dr. med. Christine Eckardt

Ärztin für Anästhesiologie

Anästhesieabteilung des Allgemeinen

Krankenhauses Altona

Paul-Ehrlich-Str. 1

2000 Hamburg 50

Dr. med. Andreas Eisenschenk

Orthopädische Klinik und Poliklinik der

FU Berlin (Oskar-Helene-Heim)

Clayallee 229

1000 Berlin 33

Dr. med. Andreas Engelhardt

Neurologische Klinik der

Universität Erlangen-Nürnberg

Schwabachanlage 6

8520 Erlangen

Dr. med. Dr. med. dent. Heinz Erpenstein Zahnarzt, Parodontologie

Hoyastr. 1

4400 Münster

Dr. med. K. Ewald

Internist

Kurt-Schumacher-Str. 26

6050 Offenbach-Bieber
Stefan Exner

Arzt

Institut für Anatomie der FU Berlin

Königin-Luise-Str. 15

1000 Berlin 33

Prof. Dr. med. Ingeborg Falck

Max-Bürger-Krankenhausbetrieb

von Berlin-Charlottenburg

Sophie-Charlotten-Str. 115

1000 Berlin 19

Prof. Dr. med. Volker Faust

Abt. Psychiatrie I der Universität Ulm

Psychiatrisches Landeskrankenhaus

Weißenau

7980 Ravensburg-Weißenau

Dr. med. H.-J. Fuchs

Oberarzt der Anästhesieabteilung des

Allgemeinen Krankenhauses Altona

Paul-Ehrlich-Str. 1

2000 Hamburg 50

Dir. u. Prof. Dr. rer. nat. Ingbert Gans

Institut für Wasser-, Boden-und Lufthygiene des Bundesgesundheitsamtes

Corrensplatz 1

1000 Berlin 33

Apotheker H. W. Geisler

Königsberger Str. 13

6239 Kriftel

Dr. med. R. Gnauck

Deutsche Klinik für Diagnostik

Fachbereich Gastroenterologie

Aukammallee 33

6200 Wiesbaden

Prof. Dr, med. R. Gossrau

Institut für Anatomie der

Freien Universität Berlin

Königin-Luise-Str. 15

1000 Berlin 33

Prof. Dr. med. Dr. rer. nat. E. H. Graul Institut für Environtologie und Nuklearmedizin der Universität Marburg

Bahnhofstr. 7

3550 Marburg/Lahn

Dr. med. W. Günther

Hals-Nasen-Ohrenarzt

Glatzer Str. 43

6430 Bad Hersfeld

Dr. phil. Dipl.-Psych. Tilmann Habermas

Fachbereich Grundlagenmedizin

Institut für Medizinische Psychologie

der Freien Universität Berlin

Habelschwerdter Allee $\mathbf{4 5}$

1000 Berlin 33

Prof. Dr. Jürgen Hacke

Hahn-Meitner-Institut für Kernforschung

Berlin GmbH, Abt. Strahlenschutz

Glienicker Str. 100

1000 Berlin 39 
Prof. Dr. Dr. Erwin J. Haeberle

Bundesgesundheitsamt

AIDS-Zentrum

Reichpietschufer 74-76

1000 Berlin 30

Prof. Dr. med. R. Häring

Direktor der Chirurgischen Klinik und

Poliklinik

Klinikum Steglitz der

Freien Universität Berlin

Hindenburgdamm 30

1000 Berlin 45

Dr. med. Michael Hammer

Abt. Rheumatologie im

Zentrum Innere Medizin und Dermatologie

der Medizinischen Hochschule Hannover

Konstanty-Gutschow-Str. 8

3000 Hannover 61

Prof. Dr. med. Dr. h.c. F. Hanefeld

Kinderklinik der Georg-August-Universität

Robert-Koch-Str. 40

3400 Göttingen

Dr. med. E. Harde

Arzt für Anästhesiologie

Anästhesieabteilung des Allgemeinen

Krankenhauses Altona

Paul-Ehrlich-Str. 1

2000 Hamburg 50

Oberarzt Dr. med. M. G. Hartmann

Tropeninstitut

Bernhardt-Nocht-Str. 74

2000 Hamburg 4

Dr. med. Klaus Hartung

Abt. Immunologie und Transfusionsmedizin im Zentrum Innere Medizin und Dermatologie der Medizinischen Hochschule Hannover

Konstanty-Gutschow-Str. 8

3000 Hannover 61

Dr. med. Thomas Hebell

Johns Hopkins University School of Medicine

Division of Molecular and Clinical

Rheumatology

617 Hunterian, 725 N. Wolfe St.

USA - Baltimore, MD 21205

Prof. Dr. med. Klaus-Peter Hellriegel

Chefarzt der II. Inneren Abteilung

des Krankenhauses Moabit

Turmstr. 22

1000 Berlin 21

Dr. med. Hans-Nikolaus Herden

Chefarzt der Anästhesieabteilung des

Allgemeinen Krankenhauses Altona

Paul-Ehrlich-Str. 1

2000 Hamburg 50

Gabriele M. Heyd

Verband der Beschäftigungs- und

Arbeitstherapeuten (Ergotherapeuten) e.V.

Mittelweg 8

7516 Karlsbad-Ittersbach
Priv.-Doz. Dr. med. Klaus Heyne

Klinik für Pädiatrie der

Medizinischen Universität zu Lübeck

Kahlhorststr. 31-35

2400 Lübeck 1

Prof. Dr, iur. Manfred Hinz

Fachbereich Rechtswissenschaft der

Freien Universität Berlin

Boltzmannstr, 1

1000 Berlin 33

Prof. Dr. med. W. Höfler

Tropenmedizinisches Institut der

Universität Tübingen

Wilhelmstraße 31

7400 Tübingen

Prof. Dr. med. Dr. med. h.c. F. Hollwich Winterthurer Str. 5

8000 München 71

Prof. Dr. med. Joachim Hüter

Chefarzt der Frauenklinik

Städtisches Krankenhaus

Weinberg 1

3200 Hildesheim

Prof. Dr. med. Konrad Hummel

Institut für Blutgruppenserologie

Postfach 880

7800 Freiburg

Anne Jung

Logopädin

Eggersallee 21

2000 Hamburg 50

Prof. Dr. med. Gabriele Kaczmarczyk Klinikum Rudolf Virchow der Freien Universität Berlin (Standort Charlottenburg) Spandauer Damm 130

1000 Berlin 19

Dr. med. B. Kniesel

Arzt für Anästhesiologie

Anästhesieabteilung des Allgemeinen

Krankenhauses Altona

Paul-Ehrlich-Str. 1

2000 Hamburg 50

Prof. Dr. rer. nat. Reinhold Knopp Institut für Klinische und Experimentelle

Nuklearmedizin der Universität Bonn Sigmund-Freud-Str. 25

5300 Bonn 1

Hans K. Koebner

Burmesterstr. $4 / \mathrm{V}$

8000 München 45

Prof. Dr. med. Detlef Kömpf

Direktor der Neurologischen Klinik

der Med. Universität zu Lübeck

Ratzeburger Allee 160

2400 Lübeck 1

Prof. Dr. med. Johannes Korporal

Fachhochschule für Sozialarbeit und 
Sozialpädagogik Berlin

Karl-Schrader-Str. 6

1000 Berlin 30

Dipl.-Phys. Martin Krämer

Landeslehranstalt für technische Assistenten

in der Medizin

Leonorenstr. 35

1000 Berlin 46

Dr. W.-R. Krause

Oberarzt der Neurologisch-Psychiatrischen

Abt. des Kreiskrankenhauses -

Poliklinik Wernigerode

DDR - 3720 Blankenburg

Kristian Kunde

Arzt

Schloßstr. 4

1000 Berlin 19

Prof. Dr. med. Jürgen Kunze

Kinderklinik der Freien Universität Berlin

und Institut für Humangenetik

Heubnerweg 6

1000 Berlin 19

Dr. med. Christoph Lang

Neurologische Klinik und Poliklinik der

Universität Erlangen-Nürnberg

Schwabachanlage 6

8520 Erlangen

Dr. med. Martin Lang

Institut für Arzneimittel des

Bundesgesundheitsamtes

Seestr. 10

1000 Berlin 65

Dr. med. Hans-Eckhard Langer

Abt. Rheumatologie im Zentrum

Innere Medizin und Dermatologie der

Medizinischen Hochschule Hannover

Konstanty-Gutschow-Str. 8

3000 Hannover 61

Prof. Dr. iur. Adolf Laufs

Dr. h. c. Université de Montpellier I

Institut für geschichtliche

Rechtswissenschaft der

Universität Heidelberg

Friedrich-Ebert-Platz 2

6900 Heidelberg

Dr. med. Herbert Lieske

Arzt für Innere Medizin,

Tropenkrankheiten

Bramfelder Chaussee 252

2000 Hamburg 71

Prof. Dr. rer. nat. Walter Maier

Institut für Medizinische Parasitologie

der Universität Bonn

Sigmund-Freud-Str. 25

5300 Bonn

Dipl.-Ing. Ingrid Maßwig

GSD Gesellschaft für Systemforschung und

Dienstleistungen im Gesundheitswesen $\mathrm{mbH}$
Facheinheit technisches Krankenhausserviceund Beratungsinstitut

Stauffenbergstr. 13-14

1000 Berlin 30

Dr. med. Wilfried Mau

Abt. Rheumatologie im Zentrum

Innere Medizin und Dermatologie der

Medizinischen Hochschule Hannover

Konstanty-Gutschow-Str. 8

3000 Hannover 61

Priv.-Doz. Dr. med. Dipl.-Psych. Rolf

Meermann

Ârztl. Dir. der Psychosomat. Fachklinik

Bombergallee 10

3280 Bad Pyrmont

Prof. Dr.-Ing. Harald Meinhold

Klinikum Steglitz der

Freien Universität Berlin

Abt. für Nuklearmedizin - Radiochemie

Hindenburgdamm 30

1000 Berlin 45

Prof. Dr. med. H.-J. Merker

Institut für Anatomie der

Freien Universität Berlin

Königin-Luise-Str. 15

1000 Berlin 33

Dr. med. Heinzpeter Moecke

Anästhesieabteilung des Allgemeinen

Krankenhauses Altona

Paul-Ehrlich-Str. 1

2000 Hamburg 50

Prof. Dr. med. K. Morgenroth

Institut für Pathologie der

Ruhr-Universität Bochum

Universitätsstraße 150

4630 Bochum-Querenburg

Dr. med. Harald Müller

Oberarzt der Klinik und Poliklinik für

Neurochirurgie

Medizinische Universität zu Lübeck

Ratzeburger Allee 160

2400 Lübeck 1

Prof. Dr. med. Reinhard Nagel

Direktor der Urologischen Klinik und Poliklinik

Klinikum Rudolf Virchow der Freien Universität Berlin (Standort Charlottenburg)

Spandauer Damm 130

1000 Berlin 19

Prof. Dr. med. Diether Neubert

Institut für Toxikologie und Embryo-

pharmakologie der Freien Universität Berlin

Garystr. 5

1000 Berlin 33

Prof. Dr. med. Bernhard Neundörfer Neurologische Klinik und Poliklinik der

Universität Erlangen-Nürnberg

Schwabachanlage 6

8520 Erlangen 
Prof. Dr. med. Gerd Offermann

Medizinische Klinik und Poliklinik

Transplantationszentrum Berlin im Klini-

kum Steglitz der Freien Universität Berlin

Hindenburgdamm 30

1000 Berlin 45

Prof. em. Dr. med. Ottomar Oppel

Hermann-Ehlers-Str. 61

5600 Wuppertal 1

Dr. med. Ch. Osmer

Anästhesieabteilung des

Allgemeinen Krankenhauses Altona

Paul-Ehrlich-Str. 1

2000 Hamburg 50

Nikolaus Pechstein

Johann-Sigismund-Str. 12

1000 Berlin 31

Priv.-Doz. Dr. med. Eckhard Petri

Städtische Krankenanstalten, Akademisches

Lehrkrankenhaus der Johannes-Gutenberg-

Universität Mainz

Dr. Ottmar-Kohler-Str. 2

6580 Idar-Oberstein

Prof. Dr. med. Detlef Petzoldt

Universitätshautklinik

Vosstr. 2

6900 Heidelberg

Christine Posselt

Krankenpflegeschule

Klinikum Steglitz der

Freien Universität Berlin

Hindenburgdamm 30

1000 Berlin 45

R. Püschel

Oberarzt der Anästhesieabteilung des

Allgemeinen Krankenhauses Altona

Paul-Ehrlich-Str. 1

2000 Hamburg 50

Dr. sc. med. Rolf Radke

Breite Straße 42 A

1000 Berlin 33

Dr. rer. nat. Dipl.-Phys. B. Ramm

Radiologische Klinik

Klinikum Rudolf Virchow der Freien Univer-

sität Berlin (Standort Charlottenburg)

Spandauer Damm 130

1000 Berlin 19

Prof. Dr. Dr. H.-H. Raspe

Oberarzt der Abt. Rheumatologie im

Zentrum Innere Medizin und Dermatologie

der Medizinischen Hochschule Hannover

Konstanty-Gutschow-Str. 8

3000 Hannover 61

Dr. med. Bernhard Riffel

Neurologische Klinik

Zentralklinikum Augsburg

Stenglinstraße

8900 Augsburg
Dr. med. Arndt Rolfs

Max-Planck-Institut für Molekulare

Genetik

Ihnestr. 73

1000 Berlin 33

Prof. Dr. Dipl.-Psych. Hans Peter Rosemeier

Fachbereich Grundlagenmedizin

Institut für Medizinische Psychologie

der Freien Universität Berlin

Habelschwerdter Allee 45

1000 Berlin 33

Dr. phil. Dr. med. Rolf Saupe

Psychiatrische Klinik der

Freien Universität Berlin

Eschenallee 3

1000 Berlin 19

Dr. rer. nat. Klaus Schaller

Institut für Chemotherapie

Bayer AG

5600 Wuppertal 1

Dr. Helmut Schmidt

Landeslehranstalt für technische Assistenten in der Medizin

Leonorenstr. 35

1000 Berlin 46

Prof. Dr. Burkhard Schneeweiß

Chefarzt der Kinderklinik

Städtisches Krankenhaus im

Friedrichshain

Leninallee 49

DDR - 1017 Berlin

Prof. Dr. med. Claus C. Schnorrenberger

Deutsches Forschungsinstitut für

Chinesische Medizin e.V.

Silberbachstr. 10

7800 Freiburg

Dr. med. A. Scholz

Anästhesieabteilung des Allgemeinen

Krankenhauses Altona

Paul-Ehrlich-Str. 1

2000 Hamburg 50

Prof. Dr. med. R. Schrage

Frauenklinik der

Eberhard-Karls-Universität Tübingen

Schleichstraße

7400 Tübingen 1

Dr. med. Hiltrudis Schütte

Albert-Schweitzer-Hospital

3410 Northeim

Stephan Schwarz

Waldstraße $\mathbf{5 7}$

1000 Berlin 21

Priv.-Doz. Dr. sc. hum. Hans-Jürgen Seelos

Lehrbeauftragter für Medizinische Informatik der Heinrich-Heine-Universität Düsseldorf Rheinisches Rechenzentrum für Kirche und Diakonie GmbH

4000 Düsseldorf 30 
Dr. med. Jochen Seidel

Abt. Immunologie und Transfusionsmedizin im Zentrum Innere Medizin und Dermatologie der Medizinischen Hochschule Hannover

Konstanty-Gutschow-Str. 8

3000 Hannover 61

Prof. Dr. med. H. M. Seitz

Institut für Medizinische Parasitologie

Sigmund-Freud-Str. 25

5300 Bonn

Priv.-Doz. Dr. med. Martin Sparmann

Ltd. Oberarzt der Orthopädischen

Klinik und Poliklinik der Freien Universität

Berlin (Oskar-Helene-Heim)

Clayallee 229

1000 Berlin 33

Prof. Dr. med. Christian P. Speer

Kinderklinik der Georg-August-Universität

Robert-Koch-Str. 40

3400 Göttingen

Prof. Dr. Jürgen Spona

I. Universitäts-Frauenklinik

Spitalgasse 23

A-1090 Wien

Prof. Dr. med. Manfred Stauber

Frauenklinik und Poliklinik des

Klinikums Rudolf Virchow der

Freien Universität Berlin

(Standort Charlottenburg)

Pulsstr. 4-14

1000 Berlin 19

OMR Prof. Dr. sc. med. Johannes Staudt

Institut für Anatomie, Bereich Medizin

(Charité) der Humboldt-Universität Berlin

Schumannstr. 20/21

DDR - 1040 Berlin

Prof. Dr. med. F. Stein

Ehem. Dir. des Pathologischen Instituts

des Klinikums Rudolf Virchow der

Freien Universität Berlin

(Standort Wedding)

Augustenburger Platz 1

1000 Berlin 65

Prof. Dr. med. Beat Steinmann

Stoffwechselabteilung

Universitäts-Kinderklinik

Steinwiesstraße 75

$\mathrm{CH}$ - 8032 Zürich

Dr. med. Michael Stimpel

Medizinische Universitätsklinik II

Ostmerheimer Str. 200

5000 Köln 91

Priv.-Doz. Dr. med. Burckhard Terwey

Mozartstraße 6

2900 Oldenburg

Dr. med. E. Thieme

Ärtin für Anästhesiologie

Anästhesieabteilung des Allgemeinen
Krankenhauses Altona

Paul-Ehrlich-Str. 1

2000 Hamburg 50

Dr. rer. nat. Johannes Voelker

Am Stein 48

2000 Hamburg 63

Dr. med. Peter Voeltz

Abt. für Anästhesie und Intensivmedizin des

Berufsgenossenschaftlichen Unfallkranken-

hauses Hamburg-Boberg

Bergedorfer Straße 10

2050 Hamburg 80

Hannelore Vogt

Arztin

Anästhesieabteilung des Allgemeinen

Krankenhauses Altona

Paul-Ehrlich-Str. 1

2000 Hamburg 50

Prof. Dr. med. Peter Wagener

Oberarzt der Abt. Rheumatologie im

Zentrum Innere Medizin und Dermatologie

der Medizinischen Hochschule Hannover

Konstanty-Gutschow-Str. 8

3000 Hannover 61

Prof. Dr. Joachim Wagner

Chefarzt der I. Medizinischen Klinik/Kardio-

logie

Krankenhaus Neukölln

Rudower Str. 48

1000 Berlin 47

Hans-Joachim Weimann

Burgemeisterstr. 59

1000 Berlin 42

Dr. rer. soc. Elmar Weingarten

Institut für Soziale Medizin der

Freien Universität Berlin

Sarrazinstr. 11-15

1000 Berlin 41

Prof. Dr. H. Werner

Abt. für Medizinische Mikrobiologie

Universität Tübingen

Silcherstr. 7

7400 Tübingen

Dr. Dr. med. Helmut Weyers †

2160 Stade

Prof. Dr. med. Ernst Wiedemann

Babenhäuser Landstr. 49

6000 Frankfurt a. M. 70

Dr. med. Klaus Wildhagen

Abt. Immunologie und Transfusionsmedizin im Zentrum Innere Medizin und Dermatologie der Medizinischen Hochschule Hannover

Konstanty-Gutschow-Str. 8

3000 Hannover 61

Prof. Dr. med. Dr. jur. Reinhard Wille Sexualmedizinische Forschungs- und Beratungsstelle 
Hospitalstr. 17-19

2300 Kiel

Dr. med. Jürgen Wollenhaupt

Abt. Rheumatologie im

Zentrum Innere Medizin und Dermatologie

der Medizinischen Hochschule Hannover

Konstanty-Gutschow-Str. 8

3000 Hannover 61

Dr. med. Jürgen Wortmann

Manteuffelstr. 10

1000 Berlin 45

Prof. Dr. med. Henning Zeidler

Leiter der Abt. Rheumatologie im

Zentrum Innere Medizin und Dermatologie der Medizinischen Hochschule Hannover

Konstanty-Gutschow-Str. 8

3000 Hannover 61
Priv.-Doz. Dr. med. H. Zilch

Orthopädische Klinik und Poliklinik der

Freien Universität Berlin

(Oskar-Helene-Heim)

Clayallee 229

1000 Berlin 33

Prof. Dr. M. Zimmermann

II. Physiologisches Institut der

Universität Heidelberg

Im Neuenheimer Feld 326

6900 Heidelberg 1

Dr, rer. pol. Angela Zink

Leiterin der Facheinheit Sozialmedizin und Epidemiologie

GSD Gesellschaft für Systemforschung und Dienstleistungen im Gesundheitswesen $\mathrm{mbH}$ Stauffenbergstr. 13-14 1000 Berlin 30

\section{Redaktion}

Dipl.-Bibl. Regina Engst

Helmut Hildebrandt, M. A.

Karin Schick, Ärztin

Helmut Schwäbl †

Thomas Spitzer, M. A.

Andreas Weimann, Arzt

Jörg Winzer

Dr. med. Christoph Zink 


\section{Staudt}

Kleiner

Anctomischer Atlas

\section{mit Begleittext}

$19,5 \times 22 \mathrm{~cm}$. 124 Seiten. Mit zahlreichen Abbildungen. 1987. Gebunden DM 29,80 ISBN 3110109603

In diesem kleinen Atlas werden 58 anatomische Graphiken und dazugehörige textliche Erläuterungen auf jeweils zwei Seiten gegenübergestellt. Die Gestaltung des Textes ist klar, übersichtlich, kurz, präzise und logisch. Mit der Kombination von Bild und Text wird einer Grundforderung der Ausbildung im Fach Anatomie entsprochen, alle Fakten und ihre funktionellen Zusammenhänge zu veranschaulichen.

Der Aufbau des Atlasses erfolgt nicht streng nach systematischen Gesichtspunkten. Es werden vor allem funktionelle Systeme und topographische Gegebenheiten einzelner Körperregionen dargestellt.

Der Atlas vermittelt gesichertes Grundwissen, ist praxisorientiert und zeigt zahlreiche interdisziplinäre Beziehungen auf.

Angehörige medizinischer Assistenzberufe, Studenten der Biologie und interessierte Laien werden hier neben einer guten bildlichen Darstellung eine Fülle von kurzen, instruktiven Informationen über die Anatomie des Menschen erhalten.

\section{de Gruyter}

